\title{
AVALIAÇÃO DA HISTERESE DE SENSORES DE GASES UTILIZADOS NA DETECÇÃO DE AROMAS ALIMENTÍCIOS
}

\author{
A. M. GRABOSKI ${ }^{1}$, S. C. BALEN ${ }^{1}$, E. GALVAGNI ${ }^{1}$, T. LAZZARI ${ }^{1}$, A. CEZARO ${ }^{1}$, J. \\ STEFFENS ${ }^{1}$ e C. STEFFENS ${ }^{1}$ \\ ${ }^{1}$ Universidade Regional Integrada do Alto Uruguai e das Missões Uri - Erechim, \\ Departamento de Engenharia de Alimentos \\ E-mail para contato: adigraboski@hotmail.com
}

\begin{abstract}
RESUMO - A determinação dos aromas se faz importante já que os mesmos são responsáveis pelos atributos de sabor global dos alimentos. Essas determinações podem ser realizadas por meio de técnicas analíticas e análises sensoriais. Porém estas técnicas se limitam quanto ao custo e ao analista, pois há uma complexidade de moléculas que compõem os aromas. Neste contexto os sensores de gás surgem como uma alternativa, pois apresentam uma resposta rápida e confiável para a detecção dos aromas nos alimentos. O presente trabalho reporta $o$ desenvolvimento de sensores de gases de baixo custo por meio da técnica de formação de trilhas utilizando papel vegetal como substrato e como camada ativa, um filme fino de polianilina (Pani) dopada com ácido clorídrico $(\mathrm{HCl})$ no estado de oxidação esmeraldina, depositado pelo método de polimerização in-situ. Foi avaliada a histerese dos sensores aos aromas de maça, morango e uva. Pode-se verificar que os sensores foram sensíveis aos aromas avaliados apresentando uma baixa histerese $(5 ; 3$ e $1,7 \%)$. Portanto os dispositivos apresentam-se muito promissores para uso na área de alimentos por apresentar um baixo custo e facilidade na obtenção.
\end{abstract}

\section{INTRODUÇÃO}

A identificação de aromas tem atraído muitas pesquisas cientificas e a atenção do setor econômico. A abordagem clássica para a avaliação das características organolépticas dos alimentos é baseada na análise sensorial, ou seja, a análise que emprega o uso dos sentidos (sabor, aroma, visão e tato) realizado por um grupo de pessoas devidamente treinado. Entretanto este método contêm muitas limitações: é um método caro, demorado, limitado a compostos que não sejam tóxicos e sofre de incoerência e imprevisibilidade devido a vários fatores humanos (Banerjee et al., 2012). Outra técnica utilizada é cromatografia gasosa, devido a complexidade da maioria dos aromas de alimentos, dificultam sua caracterização por essa técnica.

A procura por técnicas alternativas para a detecção de aromas vem crescendo atualmente. Um instrumento capaz de medir a concentração ou intensidade de odores sem as limitações inerentes ao uso de painéis humanos é altamente desejável (Gostelow et al., 2001). Nesse sentido, sensores têm sido desenvolvidos para detecção de odores, vapores e gases. Porém, os sensores disponíveis no mercado, são fabricados com semicondutores inorgânicos, como o óxido de estanho. Apesar desses sensores serem baratos, eles são pouco seletivos. 
Com isso, novas alternativas vêm sendo investigadas na área de sensores, em particular de gases, e nesse contexto moléculas orgânicas têm sido exploradas, dentre as quais se destacam os polímeros condutores (Guiseppi-elie et al., 1998). O interesse em polímeros condutores se deve ao fato de que na presença de um gás sua condutividade elétrica pode ser acentuadamente alterada, e esta mudança pode então ser precisamente detectada (Paterno e Mattoso, 1998).

A polianilina (Pani) é um dos polímeros condutores mais estudados, devido ao fato de apresentar estabilidade química em condições ambiente, facilidade de polimerização, baixo custo, fazendo com que seja economicamente atraente (Duboriz, 2014). Desse modo, a utilização da Pani como camada ativa em sensores, resulta em alta sensitividade, reversibilidade e pequeno tempo de resposta, mensurando-se a variação da condutividade elétrica em função do aumento ou da redução da concentração de aromas que se deseja medir.

Para verificar o comportamento dos sensores frente a diferentes concentrações de aroma, o estudo da histerese se torna importante. Visto que é descrita como a máxima diferença na saída do sinal, dentro de uma faixa de medidas, levando em consideração os dois caminhos determinados pelo aumento da concentração de aroma (subida) e diminuição da concentração de aroma (descida). Portanto quanto menor for a histerese mais qualidade o sensor possui.

Desse modo, o presente trabalho teve como principal objetivo a obtenção de sensores de gases de baixo custo para a detecção de diferentes aromas sintéticos, empregados na indústria de alimentos, caracterizando os sensores obtidos em relação à histerese.

\section{MATERIAL E MÉTODOS}

\subsection{Desenvolvimento dos Eletrodos}

Os eletrodos interdigitados a base de grafite foram desenvolvidos pela técnica de formação de trilhas segundo metodologia descrita por Venâncio et al. (2008). As máscaras (linhas/área) usadas na técnica de formação de trilhas (Line Patterning), foram desenhadas usando software convencional (Microsoft Publisher ${ }^{\mathrm{TM}}$ e Microsoft Paint ${ }^{\mathrm{TM}}$ ) e impressas sobre um substrato de papel vegetal utilizando uma impressora "laser jet". A imagem positiva da máscara foi desenhada no software e passada para uma imagem negativa (sendo esta máscara utilizada na impressão sobre o papel vegetal).

As dispersões aquosas de grafite (Aquadag E, Acheson Colloids Company) foram preparadas para posterior deposição e recobrimento sobre as máscaras já impressas sobre o papel vegetal com espessura $63 \mathrm{~g} / \mathrm{cm}^{3}$. O preparo desta dispersão consistiu na mistura do grafite em água deionizada na proporção 1:4 (massa/massa), sendo quatro partes de água para uma de grafite (Steffens et al, 2007).

Após o preparo da dispersão aquosa de grafite se depositou 3 gotas da suspensão de grafite sobre a máscara negativa sobre o papel vegetal e com auxílio de um "glass roller" se espalhou sobre toda superfície. Após a secagem do grafite (por aproximadamente $30 \mathrm{~min}$ ) realizou-se uma nova aplicação da solução de grafite sobre a máscara. Completada a secagem 
emergiu-se a máscara em aproximadamente $40 \mathrm{ml}$ de acetona durante 30 segundos em ultrasonificador para remoção do toner e após em $40 \mathrm{ml}$ de metil etil cetona também em ultrasonificador durante 20 segundos para remoção de toda a acetona, processo este responsável pela retirada da camada de grafite sobre o tonner impresso na máscara (Venâncio et al., 2008). E acondicionou-se em dessecador para posterior deposição do filme de polímero condutor.

\subsection{Elaboração dos Sensores de Gases por Polimerização In-Situ}

A polimerização in-situ foi realizada de acordo com o descrito por Steffens et al. (2009). O procedimento para obtenção dos sensores foi efetuado em três etapas:

$1^{\circ}$ ) Purificou-se a anilina (Aldrich, 99,5\%) por destilação à vácuo evitando-se a exposição da mesma a luz, e após destilada, a mesma foi mantida a $4^{\circ}$ :C.

$2^{\circ}$ ) Foram preparadas duas soluções: A e B para a realização do processo de polimerização. A solução A foi preparada adicionando-se $198 \mu 1$ de anilina destilada em 66 $\mathrm{mL}$ de Ácido Clorídrico ( $\mathrm{HCl})(1 \mathrm{M})$ e a solução B foi preparada adicionando-se 0,498 gramas de persulfato de amônio (APS, Merck, 98\%), em $33 \mathrm{~mL}$ de $\mathrm{HCl}$ (Merck, 99\% ) (1M). Acondicionou-se as soluções A e B em banho de gelo até atingirem a temperatura de $0^{\circ} \mathrm{C}$.

$3^{\circ}$ ) Os eletrodos foram fixados em uma folha de politereftalato de etileno (PET), onde foi disposta no interior de um béquer, que se encontrava dentro de um banho de gelo. Adicionou-se a solução A e B dentro do béquer que continha os eletrodos interdigitados. A temperatura do sistema foi controlada para que a reação ocorre-se a $0^{\circ} \mathrm{C}$, sob agitação magnética (ocorreu então a reação de polimerização da anilina onde a mesma foi dopada por protonação e obteve-se o polímero no estado de oxidação esmeraldina). Após 100 minutos de reação, retirou-se os sensores do béquer e lavou-se com $\mathrm{HCl}(1 \mathrm{M})$ em abundância para retirada do excesso da solução nos sensores. Em seguida, acondicionou-se os sensores em dessecador a vácuo até completa secagem.

\subsection{Análise da Histerese dos Sensores de Gases aos Aromas}

Para avaliar a histerese dos sensores de gases com filme de Pani dopados com $\mathrm{HCl}$, os aromas comerciais de maça, morango e uva (Duas Rodas), foram diluídos em água destilada em diferentes concentrações $(0,20,40,60,80$ e 100\%).) os quais foram escolhidos pois são comumente utilizados a indústria de alimentos (balas, confeitos, iogurtes). Podendo assim gerar estudos de aplicação na indústria de alimentos. Para realização dos experimentos um aparato experimental foi construído. O sensor de gás foi fixado no interior da câmara e, inicialmente, uma linha de base foi obtida medindo-se a resistência elétrica dos sensores a cada $1 \mathrm{~min}$ durante $15 \mathrm{~min}$. Esta medida foi obtida por meio de um multímetro (FLUKE, modelo 77III). Também em cada análise monitorou-se a temperatura e a umidade com o auxílio de um sensor termo-higrômetro (IMIMIPA MT-241), para verificar a estabilidade das mesmas para que não influenciassem na resposta. Após aquisição de linha base, os sensores foram expostos aos aromas, para obtenção das curvas de histerese onde foram avaliados dois caminhos de subida (aumentando a concentração de aroma, de 0 a 100\%) e descida (diminuindo a concentração de aroma, de 100 a $0 \%$ ), onde novamente foi medida a resistência 
a cada minuto durante 5 minutos. Cada teste de histerese com os aromas foram feitos separadamente obtendo-se assim três curvas de histerese.

\section{RESULTADOS E DISCUSSÕES}

\subsection{Histerese}

A Figura 1 apresenta a análise de histerese dos sensores de gases com filme de Pani aos aroma de maçã (A), morango (B) e uva (C) respectivamente.

A histerese pode ser descrita como a máxima diferença na saída do sinal, dentro de uma faixa de medidas, levando-se em consideração dois caminhos (o de subida e o de descida). A histerese pode ser descrita como a máxima diferença na saída do sinal, dentro de uma faixa de medidas, levando-se em consideração dois caminhos (o de subida e o de descida). As curvas foram realizadas a uma temperatura de $\pm 25^{\circ} \mathrm{C}$ e umidade relativa de $58 \%$.

Figura 1. Análise de histerese dos sensores de gases aos aromas de: maçã (A), morango (B) e uva (C) em diferentes concentrações.
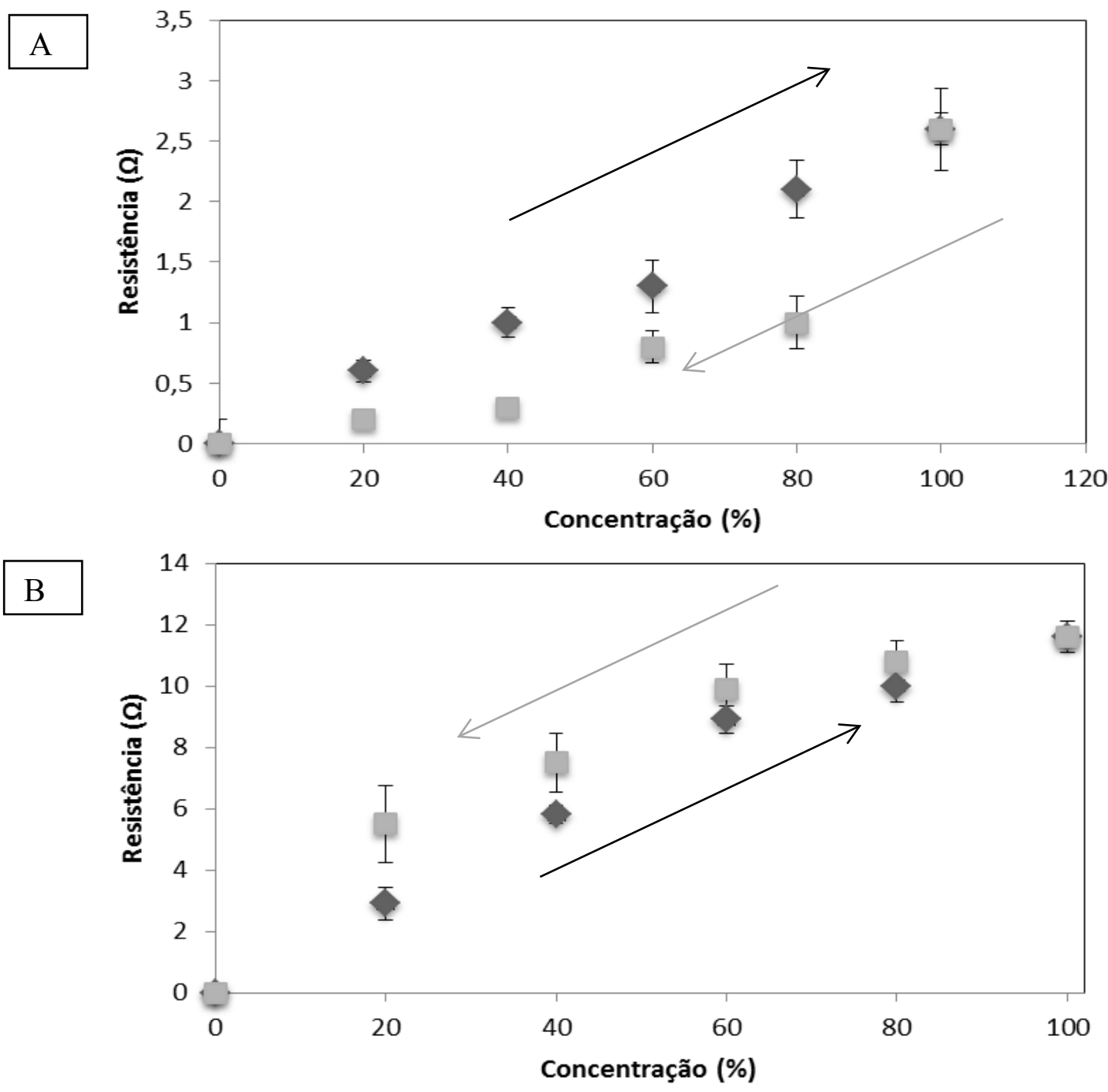


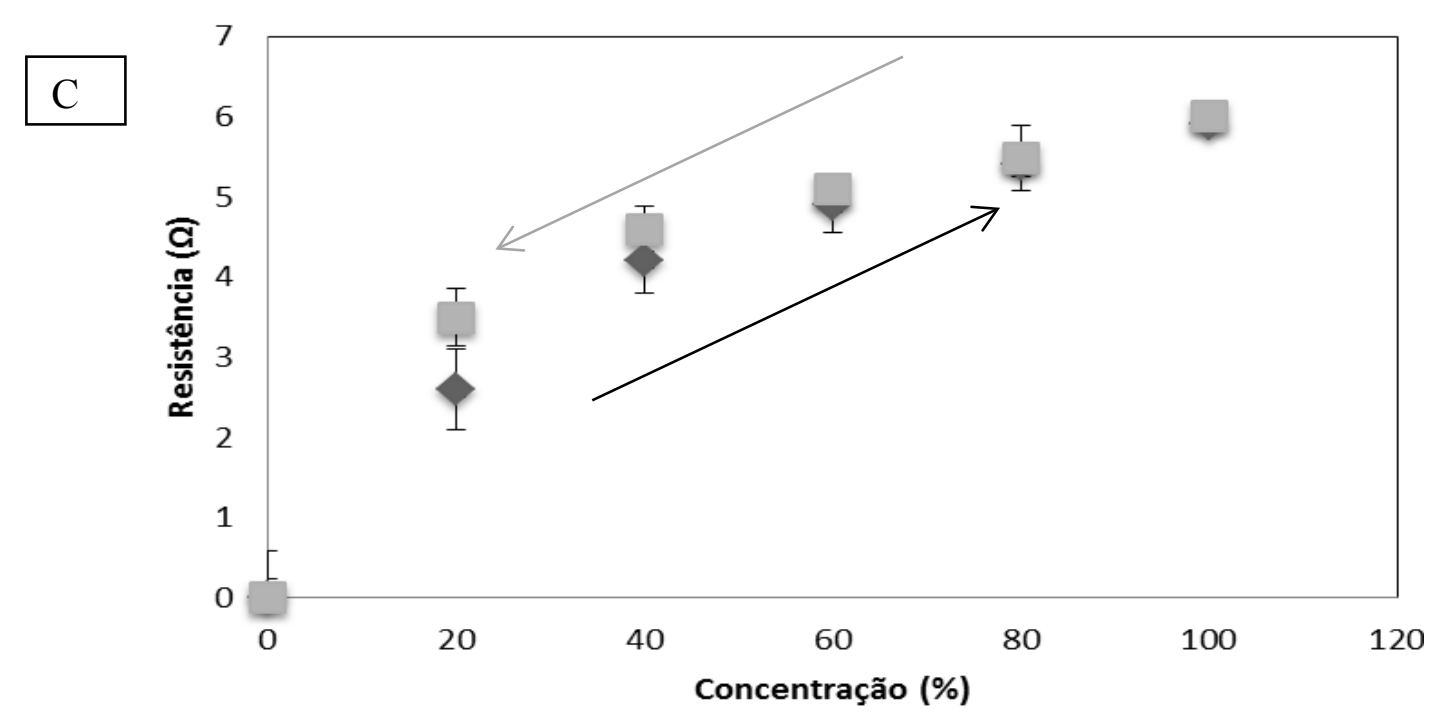

Os resultados demonstraram que os sensores de gases apresentaram histerese de 5\%, 3\% e 1,7 \% para os aromas de maçã, morango e uva, respectivamente. Portanto pode se dizer que os sensores apresentaram uma pequena histerese, o que se repete com a maioria dos sensores comerciais, ou seja, os sinais para o aumento e diminuição diferem pouco em certas medidas.

Esta pequena histerese pode ser explicada pela condutividade da Pani que é conhecida por possuir histerese, o que foi verificado na concentração versus resistência, características de respostas do sensor de gás, onde a curva no sentido positivo apresenta uma pequena alteração em relação a variação no sentido inverso. Esta existência de histerese é mais estreitamente relacionada com o nível de dopagem da polianilina do que o $\mathrm{pH}$. Esta histerese aparente ou "efeito de memória" tem sido atribuída a flexibilização estruturais (Lee et al., 2010; He et al., 2001).

Cankurtaran et al. (2013) avaliaram a histerese para o sensor de ácido sulfônico difenilamina e polietileno glicol em relação a umidade relativa e verificaram que o máximo de histerese durante a subida e descida de umidade foi de menos de 3\%. Steffens et al. (2012) avaliaram a histerese em sensores de cantilever funcionalizados com filme fino de polianilina em umidade e verificou $2,82 \%$ de histerese a $20,0{ }^{\circ} \mathrm{C}$. . Ma et al. (2008) verificaram que sensores elaborados com polímeros estão sujeitos a exibir problemas de histerese, devido à tendência das moléculas de água adsorvidas formarem ligações de hidrogênio com a matriz polimérica. Uma vez que as moléculas de água se ligam com a matriz polimérica, quando a umidade for diminuída, certo número de moléculas permanecerá ligada ao polímero, em vez de serem dessorvidas. Visto que as moléculas de água são mantidas no polímero, as características básicas do sensor são alteradas, causando uma histerese. Comparando o resultado destes estudos, pode-se verificar que as histereses dos sensores de gás obtida no presente estudo foram relevantes com os diferentes aromas alimentícios avaliados.

\section{CONCLUSÃO}

Os sensores de gases com filme de Pani desenvolvidos foram sensíveis aos aromas de morango e maçã e uva apresentando uma baixa histerese. Este estudo sugere que os sensores 
de gases que utilizam como substrato o papel vegetal são de baixo custo, flexíveis e de materiais altamente sensíveis para a aplicação no sensoriamento de aromas alimentícios.

\section{REFERÊNCIAS}

BANERJEE R.; TUDU B.; SHAW L.; JANA A.; BHATTACHARYYA N.; BANDYOPADHYAY R. Instrumental testing of tea by combining the responses of electronic nose and tongue. J Food Eng, v.110, p. 356-363. 2012.

CANKURTARAN, H.; YAZICI, O.; DINC S.; KARAMAN, F. Humidity Sensitive Properties of Electronically Conductive Poly(diphenylamine sulfonic acid) and Its Block Copolymer and Blends. Int. J. Electrochem. Sci., v. 8, p. 3265 - 3278, 2013.

DUBORIZ, I.; PUD A. Polyaniline/poly(ethylene terephthalate) film as a new optical sensing material. Sensor Actuat, v. 190, p. 398-407, 2014.

GOSTELOW, P.; PARSONS, S.A.; STUETZ, R.M. Odour measurements for sewage treatment works. Water Research, v. 35, n. 3, p. 579-597, 2001.

GUISEPPI-ELIE A.G.; WALLACE G.G.; MATSUE T.; in: T.A. Skotheim, R.L. Elsenbaumer, J.R. Reynolds (Eds.). Handbook of Conducting Polymers, Marcel Dekker, New York, p. 963, 1998.

HE, H.; ZHU, J.; TAO, N.J.; NAGAHARA, L.A.; AMLANI, I.; TSUI, R. A conducting polymer nanojunction switch. J. Am. Chem. Soc. v. 123, p. 7730-7731, 2001.

LEE, S.-Y.; LEE, S.-K.; LIM, H.; CHOI, G.-R. Irreversible degradation behaviors of an electrolyte-gated polyaniline (PANI) nanowire field-effect transistor. J. Korean Phys. Soc., v. 57, p. 1416-1420, 2010

MA, Q.; TIPPING, R.H.; LEFORESTIER, C. Temperature dependences of mechanisms responsible for the water-vapor continuum absorption: 1. Far wings of allowed lines. J. Chem. Phys, v. $128,2008$.

PATERNO, G. L; MATTOSO, L. H. Novos materiais poliméricos para sensores de gases. Comunicado Técnico Embrapa, 1998.

STEFFENS C., CORAZZA M. L., FRANCESCHI E., CASTILHOS F., HERRMANN P. S. P., Oliveira, J. V. Development of gas sensors coatings by polyaniline using pressurized fluid. Sensor Actuat, v. 171-172, p. 627-633, 2012.

STEFFENS, C.; BRITTO, D.; HERRMANN, P.S.P. Chitosan films as substrate to line patterning technique of graphite (LPTG). Application as sensors. Abstract $N^{\circ}$. MM3.14. Boston, MA: Mater Res Society 2007.

STEFFENS, C.; MANZOLI, A.; FRANCHESCHI, E.; CORAZZA, M. L.; CORAZZA, F. C.; OLIVEIRA, J. V.; HERRMANN, P.S.P. Low-cost sensors developed on paper by line patterning with graphite and polyaniline coating with supercritical $\mathrm{CO}_{2}$. Synth. Metals, v. 159 p. 2329-2332, 2009.

VENÂNCIO, E.V.; MATTOSO, L.H.C.; HERRMANN, P. S.P; MACDIARMID, A. G. Line patterning of graphite and the fabrication of cheap, inexpensive, "throw-away" sensors. Sensor Actuat v.130, p. 723-729, 2008. 